\title{
Squamous Cell Carcinoma of the Descending Colon: Report of a Case and Literature Review
}

\author{
Hidenori Miyamoto ${ }^{a}$ Masanori Nishioka ${ }^{a} \quad$ Nobuhiro Kurita $^{a}$ \\ Junko Honda ${ }^{a}$ Kouzou Yoshikawa ${ }^{a}$ Jun Higashijima ${ }^{a}$ \\ Tomohiko Miyatani $^{a}$ Yoshimi Bandou ${ }^{b}$ Mitsuo Shimada ${ }^{a}$ \\ Departments of a Digestive Surgery and ${ }^{b}$ Molecular and Environmental Pathology, \\ Institute of Health Biosciences, The University of Tokushima Graduate School, \\ Tokushima, Japan
}

\section{Key Words}

Squamous cell carcinoma $\cdot$ Colon $\cdot$ Prognosis

\begin{abstract}
It is very rare that squamous cell carcinoma (SCC) arises from colorectal epithelium. An 89-year-old man was treated in 2001 with chief complaints of anorexia, abdominal pain, and low grade fever. The histological diagnosis as SCC was determined by biopsy during a colonoscopy. We diagnosed primary SCC of the colon because except in the colon no malignant lesions were found by systemic CT. Surgical complete resection was performed. However, he died three months after surgical resection because of hepatic metastasis and cachexia. The prognosis of this disease seems to be worse than that of adenocarcinoma.
\end{abstract}

\section{Introduction}

It is well known that more than $90 \%$ of colorectal diseases are adenocarcinoma, with the majority of remaining cases having no epithelial histology such as carcinoid tumors, sarcomas, and lymphoid tumors [1]. Pure squamous cell carcinoma (SCC) is not uncommon in glandular organs such as the uterus, lung, and pancreas, but a tumor of the intestinal tract is rare [2]. The incidence of SCC of the colon and rectum has been reported to be 0.25 to 0.1 per 1,000 colorectal carcinomas [3]. After the first case report in 1919 [4], a total 72 pure SCCs of the colon and rectum have been reported [3, 5-7]. Clinical characteristics, biologic behavior, and treatment response of this colorectal cancer are largely unknown. In this paper we report a case of primary SCC of the descending colon. 


\section{Case Report}

An 89-year-old man underwent surgical operation for sigmoid colon cancer in 1994. Histological feature was well differentiated adenocarcinoma. He visited our hospital with low grade fever, anorexia and abdominal pain, seven years after the first operation in May 2001. Abdominal examination revealed a mass in the left upper quadrant. Barium enema showed irregular stenosis of the colon at the splenic flexure. Abdominal computed tomography (CT) showed wall thickness and stenosis of the colon at the splenic flexure and lymph node enlargement around the tumor (fig. 1). Colonoscopy revealed stenosis with ulceration in the colon at the splenic flexure. The histological diagnosis of SCC was determined by biopsies during colonoscopy. Although chest, neck and cervical CT were done, tumors were only identified in the colon. Therefore, we concluded that the colon was the primary lesion site. Although this tumor was a huge mass which penetrated the jejunum and adhered to the left kidney and left diaphragm, left hemicolectomy, partial resection of the jejunum and splenectomy were performed. The resected mass was huge, $11.0 \times 8.0 \mathrm{~cm}$, with penetration to the jejunum (ig. 2). Pathology demonstrated SCC of the colon at the splenic flexure invading the jejunum, diaphragm and capsule of the kidney (fig. 3a). Regional lymph nodes had metastasis of SCC (fig. 3b). A curative operation was performed. Adjuvant chemotherapy was not started because of the advanced age of the patient. Three months after the operation he died because of multiple liver metastases and cachexia.

\section{Discussion}

SCCs of the colon are an extremely rare clinical entity. The first case of a pure SCC of the colon was reported in the German literature by Schmidtmann in 1919 [4]. In Japan, Murakami et al. reported the first case of a pure SCC of the colon in 1974 [8]. Since that initial description 72 cases of pure SCCs of the colon and rectum have been reported (table 1) [3, 5-7].

Certain criteria must be satisfied before a diagnosis of primary SCC of the colon is made [9]. First, metastasis from other sites to the bowel must be ruled out. Second, a squamous-lined fistulous tract must not involve the affected bowel, because this may be a source of SCCs. Third, SCCs of the anus with proximal extension must be excluded. Fourth, SCC must be confirmed by histological analysis. Our case satisfied all these criteria.

The prognosis of patients with colorectal SCC is difficult to establish because of the rarity of these tumors. The colorectal SCC seems to be more frequently locally invasive and more likely to involve regional lymphatics than the adenocarcinomas, probably because of a delayed diagnosis. In this case, the tumor was pT4 (invasion of the diaphragm and capsule of the kidney) and lymph node involvement. However, curative resection with a negative resection margin was performed. Comer et al. [10] suggested a poorer prognosis for patients with colorectal SCC than adenocarcinoma.

The role of adjuvant chemotherapy or radiation remains unknown. Gelas et al. [3] reported that surgical resection after neoadjuvant combination of chemotherapy and external beam radiation therapy was useful for rectal SCC. Juturi et al. [5] reported that combination of cisplatin, 5-fluorouracil, and leucovorin would be a possible treatment option for patients with metastatic colorectal SCC. Copur et al. [7] reported that cisplatin, etoposide and 5-fluorouracil combination chemotherapy was effective and serum SCC antigen level was a useful marker of response to chemotherapy. Chemotherapy for colorectal SCC has been controversial. Nowadays, we think that surgical resection may be the first choice and adjuvant treatment (chemotherapy or radiation therapy) may be done if the patient has a good performance status.

In conclusion, advanced colorectal SCC with invasion to adjacent organs and metastatic lymph nodes had a poor prognosis. Treatment selection is difficult because 
colorectal SCC is a very rare disease. However, surgical resection and adjuvant chemotherapy is a better approach to the treatment of colorectal SCC.

Table 1, Squamous cell carcinoma of the colon and rectum: clinical feature

\begin{tabular}{|c|c|c|c|c|c|c|}
\hline Case & Author (year) & $\begin{array}{l}\text { Age } \\
\text { years }\end{array}$ & $\begin{array}{l}\text { Gen- } \\
\text { der }\end{array}$ & Location & Treatment & Outcome \\
\hline 1 & Schmidtmann (1919) & 65 & M & cecum & & DOD at 1 month \\
\hline 2 & Catell and Williams (1943) & 63 & M & rectum at $10 \mathrm{~cm}$ & surgical resection & alive at 3.5 years \\
\hline 3 & Hicks and Cowling (1955) & 90 & $\mathrm{~F}$ & ascending colon & N/A & DOD at 1 month \\
\hline 4 & Wiener et al. (1962) & 52 & $\mathrm{~F}$ & rectum at $9 \mathrm{~cm}$ & APR & died at 1 year \\
\hline 5 & Larizaden and Powell (1965) & 44 & $\mathrm{~F}$ & hepatic flexure & right hemicolectomy & alive at 8 months \\
\hline 6 & Wood (1967) & 58 & M & cecum & right hemicolectomy & N/A \\
\hline 7 & Minkowitz (1967) & 49 & $\mathrm{~F}$ & rectosigmoid & proctocolectomy & dead at 5 months \\
\hline 8 & Gaston (1967) & 65 & M & cecum & right hemicolectomy & alive at 2 years \\
\hline 9 & Pemberton and Lendrum (1968) & 48 & $\mathrm{~F}$ & ascending colon & right hemicolectomy & alive at 2 years \\
\hline 10 & Birnbaum (1970) & 82 & M & ascending colon & right hemicolectomy & N/A \\
\hline 11 & Comer et al. (1971) & 34 & $\mathrm{~F}$ & rectum at $8 \mathrm{~cm}$ & APR & alive at 13 years \\
\hline 12 & & & & transverse colon & & \\
\hline 13 & & & & descending colon & & \\
\hline 14 & & & & upper rectum & & \\
\hline 15 & & & & ascending colon & & \\
\hline 16 & & & & hepatic flexure & & \\
\hline 17 & & & & sigmoid & & \\
\hline 18 & & & & sigmoid & & \\
\hline 19 & Lewis et al. (1971) & 61 & M & cecum & right hemicolectomy & dead at 10 days \\
\hline 20 & Balfour (1972) & 63 & M & sigmoid & & alive at 18 months \\
\hline 21 & Horne and McCulloch (1978) & 53 & M & cecum & right hemicolectomy & dead at 11 months \\
\hline 22 & Crissman (1978) & 72 & M & transverse colon & colectomy & dead at 3 days \\
\hline 23 & Burgess et al. (1979) & 43 & M & hepatic flexure & right hemicolectomy & dead at 1 year \\
\hline 24 & Williams et al. (1979) & N/A & N/A & rectum & N/A & N/A \\
\hline 25 & Kahn et al. (1979) & 64 & M & ascending colon & N/A & N/A \\
\hline 26 & Hickey and Corson (1981) & 48 & $\mathrm{~F}$ & transverse colon & $\begin{array}{l}\text { transverse/left hemi- } \\
\text { colectomy }\end{array}$ & alive at 21 months \\
\hline 27 & Petrelli et al. (1981) & 73 & M & sigmoid & palliative colostomy & dead at 9 days \\
\hline 28 & Pitella and Torres (1982) & 33 & M & ascending colon & ileocolic bypass & dead at 10 days \\
\hline 29 & Hey and Brandt (1982) & N/A & N/A & colon (not specified) & N/A & N/A \\
\hline 30 & & N/A & N/A & colon (not specified) & N/A & N/A \\
\hline 31 & Lyttle (1983) & 65 & $\mathrm{~F}$ & ascending colon & right hemicolectomy & alive at 2 months \\
\hline 32 & Vezeridis et al. (1983) & 56 & M & rectum at $10 \mathrm{~cm}$ & APR & intraoperative death \\
\hline 33 & & 57 & M & transverse colon & colectomy & alive at 14 months \\
\hline 34 & & 44 & M & rectum & APR & dead at nine days \\
\hline 35 & & 61 & $\mathrm{~F}$ & rectum & $\begin{array}{l}\text { investigational } \\
\text { chemotherapy }\end{array}$ & dead at 4 months \\
\hline 36 & & 66 & $\mathrm{~F}$ & rectum at $5 \mathrm{~cm}$ & 5-FU and radiation & dead at 15 months \\
\hline 37 & & 62 & $\mathrm{~F}$ & rectum & APR & dead at 13 months \\
\hline 38 & Gould et al. (1983) & 61 & M & splenic flexure & ileocolic bypass & dead at 3 months \\
\hline 39 & Francioni et al. (1983) & N/A & N/A & colon (not specified) & N/A & N/A \\
\hline 40 & Forouhar (1984) & N/A & N/A & colon (not specified) & N/A & N/A \\
\hline 41 & Balsano (1985) & 65 & M & cecum & right hemicolectomy & N/A \\
\hline 42 & & 58 & M & ascending colon & right hemicolectomy & N/A \\
\hline 43 & Chulia et al. (1986) & & & hepatic flexure & & \\
\hline 44 & Navarro et al. (1986) & & & colon & & \\
\hline 45 & & & & colon & & \\
\hline 46 & & & & colon & & \\
\hline 47 & Pigott and Williams (1987) & & & rectum & APR & doing well \\
\hline 48 & Shao et al. (1987) & & & ascending colon & right hemicolectomy & \\
\hline 49 & Lundquest et al. (1988) & & & cecum & & \\
\hline 50 & McMahon (1991) & & $\mathrm{F}$ & transverse colon & & \\
\hline
\end{tabular}


51 Wyatt (1991)

52 Schneider et al. (1992)

53

54

55 Betancourt et al. (1992)

56 Vignale (1993)

57 Yoshida et al. (1994)

58 Vraux et al. (1994)

59 Alekseev et al. (1994)

60 Morita (1995)

61 Petrelli et al. (1996)

63 Juturi et al. (1998)

64

$\begin{array}{ll}65 & \text { Goodfellow et al. (1999) } \\ 66 & \text { Copur et al. (2001) } \\ 67 & \text { Gelas et al. (2002) } \\ 68 & \\ 69 & \\ 70 & \\ 71 & \\ 72 & \\ 73 & \text { our case }\end{array}$

$71 \quad \mathrm{M}$

cecum

rectum

rectum

rectum

hepatic flexure

69 M sigmoid colon

51 M splenic flexure

colon

colon

$57 \quad M \quad$ ascending colon

$62 \mathrm{M}$ rectum

41 F cecum

61 F hepatic flexure

$66 \mathrm{M}$

hepatic flexure

$54 \quad \mathrm{M}$

$47 \quad \mathrm{~F}$

$63 \quad \mathrm{M}$

$70 \quad \mathrm{~F}$

93

45

43

89 rectosigmoid

rectum

rectum

rectum

rectum

rectum

rectum

descending colon surgery and RT

surgery and RT

surgery and RT

left hemicolectomy

chemotherapy

APR

colectomy

right hemicolectomy

alive NED

18 years after

diagnosis

left hemicolectomy and $\mathrm{CT}$

dead of disease

15 months

after diagnosis

right hemicolectomy

N/A

$\mathrm{APR}+\mathrm{CT}$

dead at 18 months after diagnosis

$\mathrm{APR}+\mathrm{CT}$

$\mathrm{APR}+\mathrm{CT}$

APR

RT

low anterior resection

low anterior resection

descending colectomy dead 3 months after operation

$\mathrm{N} / \mathrm{A}=$ Not available; $\mathrm{DOD}=$ died of disease; $\mathrm{APR}=$ abdominoperineal resection; 5 -FU = 5-fluorouracil; $\mathrm{RT}=$ radiation therapy; $\mathrm{CT}=$ chemotherapy; $\mathrm{NED}=$ no evidence of disease. 


\begin{tabular}{c|l|l|l}
$\begin{array}{c}\text { CaseReportsin } \\
\text { Gastroenterology }\end{array}$ & $\begin{array}{l}\text { Case Rep Gastroenterol 2007;1:77-83 } \\
\text { D0I: 10.1159/000107470 }\end{array}$ & Published online: August 29, 2007 & $\begin{array}{l}\text { O 2007 S. Karger AG, Basel } \\
\text { ISSN 1662-0631 } \\
\text { www.karger.com/crg }\end{array}$ \\
\hline
\end{tabular}

Fig. 1. Abdominal computed tomography (CT) scan showed a large heterogeneous mass involving the colon at the splenic flexure and swelling lymph nodes.

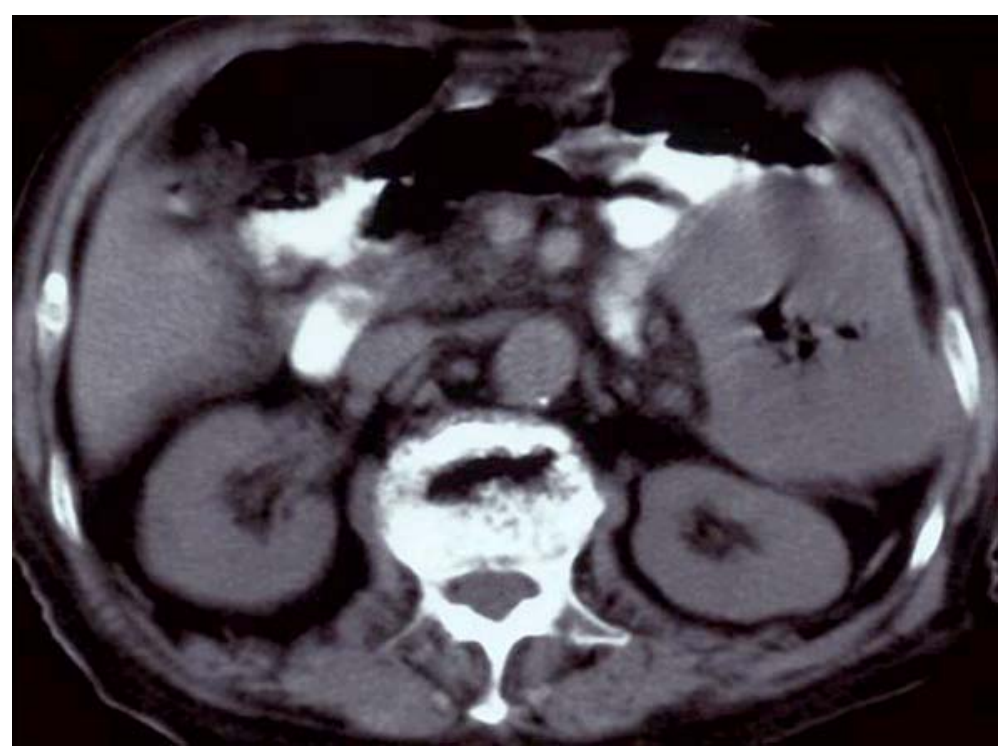

Fig. 2. Macroscopic appearance. The resected specimen was a huge mass of $11.0 \times 8.0 \mathrm{~cm}$.

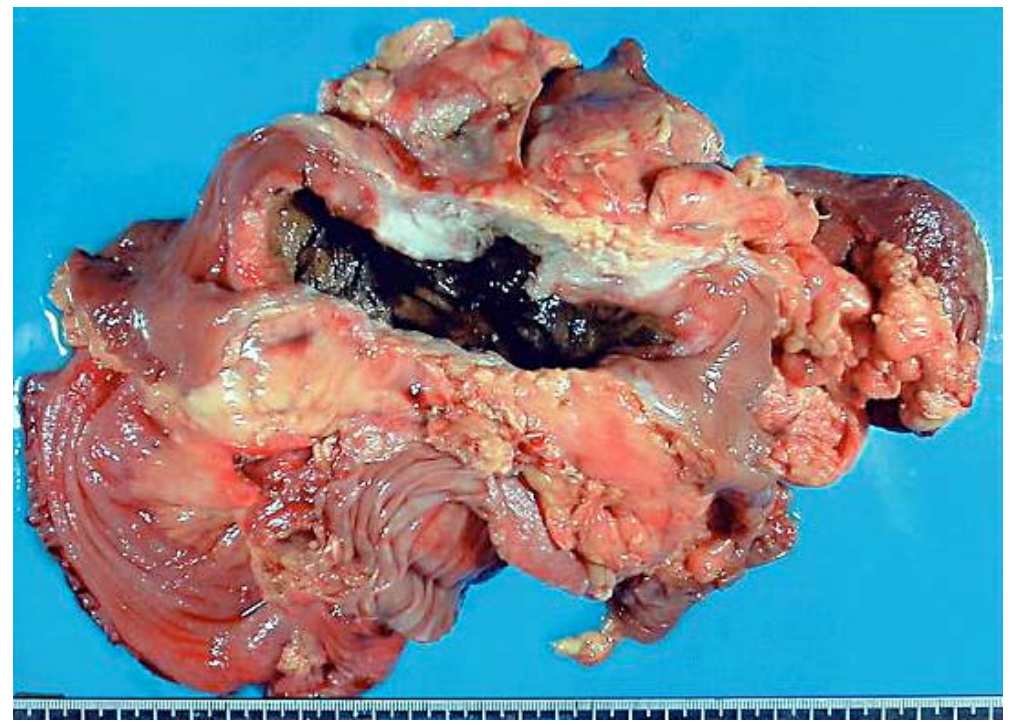




\begin{tabular}{c|l|l|l}
$\begin{array}{c}\text { CaseReportsin } \\
\text { Gastroenterology }\end{array}$ & $\begin{array}{l}\text { Case Rep Gastroenterol 2007;1:77-83 } \\
\text { D0I: 10.1159/000107470 }\end{array}$ & Published online: August 29, 2007 & $\begin{array}{l}\text { O 2007 S. Karger AG, Basel } \\
\text { ISSN 1662-0631 } \\
\text { www.karger.com/crg }\end{array}$ \\
\hline
\end{tabular}

Fig. 3. a Primary tumor showing weak squamous change, demonstrating moderately differentiated SCC. Original magnification $\times 100$. HE stain. b Metastatic lymph node showing metastatic, moderately differentiated SCC. Original magnification $\times 100$. HE stain.
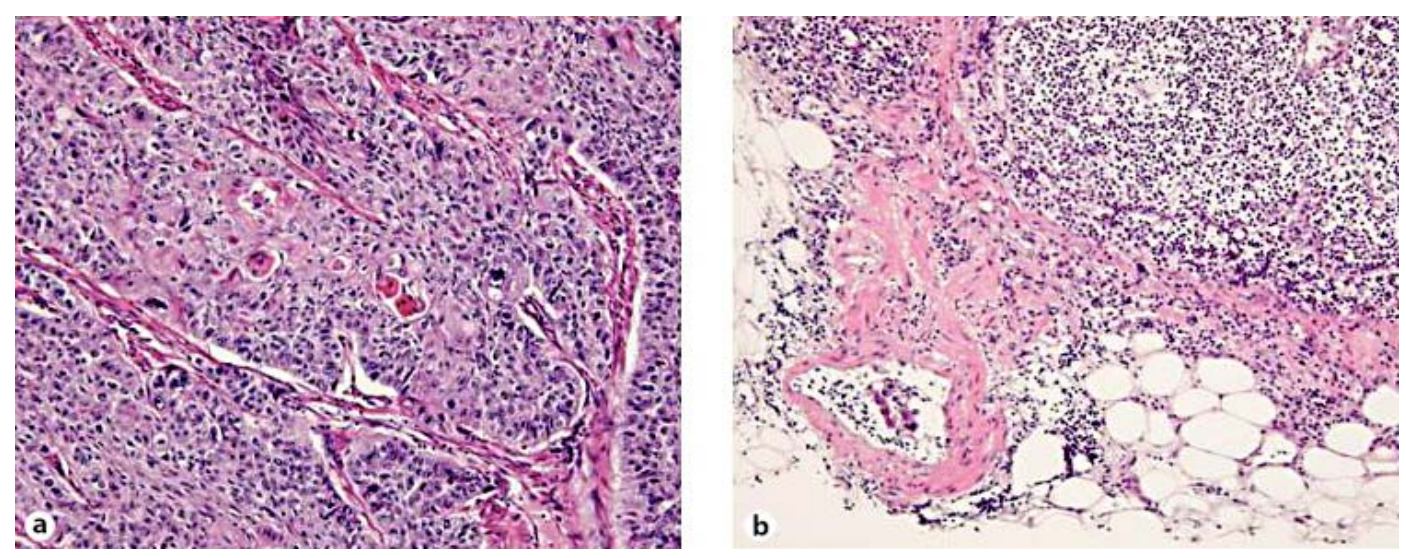


\section{References}

1 Morson BC, Sobin LH: Histologic typing of intestinal tumors: WHO technical report 15. Geneva, World Health Organization, 1976.

2 Novak ER, Wooddruff JD: Gynecologic and obstetric pathology, ed 5. Philadelphia, WB Saunders, 1962.

3 Gelas T, Peyrat P, Francois Y, et al: Primary squamous-cell carcinoma of the rectum. Dis Colon Rectum 2002;45:1535-1540.

4 Schmidtmann M: Zur Kenntnis seltener Krebsformen. Virch Arch Pathol 1919;226:100-118.

5 Juturi JV, Francis B, Koontz PW, et al: Squamous-cell carcinoma of the colon responsive to combination chemotherapy. Dis Colon Rectum 1999;42:102-109.

6 Goodfellow PB, Brown SR, Hosie KB, et al: Squamous cell carcinoma of the colon in an asbestos worker. Eur J Surg Oncol 1999;25:632-633.

7 Copur S, Ledakis P, Novinski D, et al: Squamous cell carcinoma of the colon with an elevated serum squamous cell carcinoma antigen responding to combination chemotherapy. Clin Colorectal Cancer 2001;1:55-58.

8 Murakami H, Miyagi S, Satoh T, et al: A case of squamous cell carcinoma of the sigmoid colon (in Japanese). Geka Shinryou (Surgical Diagnosis and Treatment) 1974;16:422-425.

9 Williams GT, Blackshaw AJ, Morson BC: Squamous carcinoma of the colorectum and its genesis. J Pathol 1979;129:139-147.

10 Comer TP, Beahrs OH, Dockerty MB: Primary squamous cell carcinoma and adenoacanthoma of the colon. Cancer 1971;58:111-117. 